\title{
Three-Dimensional Planar Metallic Lenses Based on Concentric Rings with Modulated Subwavelength Width
}

\author{
Di Feng ${ }^{1,2}$, Chunxi Zhang ${ }^{1}$, Lishuang Feng ${ }^{1}$, Yuanhong Yang ${ }^{1}$ \\ ${ }^{1}$ School of Instrumentation Science and Optoelectronics Engineering, Beihang University, Beijing, China; ${ }^{2}$ State Key Laboratory of \\ Millimeter Waves, Southeast University, Nanjing, China. \\ Email: fengdi@buaa.edu.cn
}

Received October $7^{\text {th }}, 2012$; revised November $6^{\text {th }}, 2012$; accepted November $25^{\text {th }}, 2012$

\begin{abstract}
A kind of Subwavelength Planar Metallic Lenses (SPMLs) is proposed to realize far-field optical focusing in the visible range based on concentric rings with modulated width in a silver film. The width of each metallic ring is mutative so that the radiation fields of surface plasmon polaritons can be controlled and the relevant phase retardations can be modulated to make a beam focus at the desired position. For comparison, the Subwavelength Planar Dielectric Lenses (SPDLs) structured on silica glass with the same concentric ring shapes as SPMLs are analyzed, although without opaque metal coating on SPDLs, the computational results show that SPMLs can support higher intensity focal spot, narrower full-width half-maximum beam width, and longer depth of focus at the focal region under certain lens thickness due to the coupling of surface plasomon polaritons, diffracted evanescent waves and propagated electromagnetic waves.
\end{abstract}

Keywords: Subwavelength Structures; Focus; Surface Plasmons; Diffractive Lenses

\section{Introduction}

Metallic nanostructures have been a subject of considerable interest in recent years, since the discovery of extraordinary optical transmission phenomena through subwavelength metallic aperture array [1,2]. Such light transmission phenomena may be explained as an excitation of a Surface Plasmon Polaritons (SPPs) mode which is an electromagnetic excitation existing on the surface of noble metals at the nano-scale aperture entrance where electromagnetic wave propagates through it before emitting into radiation modes at the exit [3-5]. Otherwise, with developments of nanofabrication techniques, such as laser beam writing, electron beam lithography, and focused ion beam etching, nano-scale metallic structures with high refinement are available. This has enabled to develop new nanophotonic integrated devices based on the plasmonic behavior of metals used for wave guiding, biosensing, and superfocusing, etc. [6,7]. In general cases, light can be focused by dielectric optical elements, such as refractive lenses, diffractive lenses, etc, but due to the diffraction of light wave, the resolution of a dielectric lens is limited to about half of the working wavelength. Driven by the potential applications in higher resolution imaging and microscopy, higher density optical data storage, and optoelectronics integrated devices, etc, and by the discovery of extraordinary optical transmission phenomena, researchers have paid more attentions to the study of plasmonic lenses or metallic lenses based on SPPs and near field evanescent waves, due to the fact that the lens provides a possible solution to get a higher resolution, which also opens up a new way for new kinds of nano-optics devices with thin metallic film. Recently, metal coated Fresnel Zone Plates (FZPs) have been proposed to realize superlens in the visible wavelength [8-10], otherwise with constant depth but variant widths, say, planar metallic lens, is proposed to focus beam by modulating light phase [11], and more, the same group found that the relative phase of emitting light scattered by surface plasmon in a single subwavelength metallic groove can be modulated by the groove depth [12]. Lieven, et al. experimentally demonstrated planar lenses based on nanoscale slit arrays in a metallic film, and got an excellent agreement between electromagnetic simulations of the design and confocal measurements on manufactured structures [13]. But subwavelength structure sizes of lenses mentioned above are quite small (less then several decades nanometers for some slits or rings), made in a thick metal film (hundreds of nanometers), which is difficult to fabricate. Although, more recently, Chen has proposed a new plasmonic lens with refractive index modulation technology, and gotten a higher light intensity at the focus 
[14], this method still increases the burden for design and fabrication of lenses. In our previous study, we analyzed focusing characteristics of one-dimensional planar dielectric lenses with binary subwavelength structures based on the electromagnetic theory in detail [15]. This Subwave-length Planar Dielectric Lenses (SPDLs) can focus light beam quite well and satisfy the planar micro or nano lithographic process. Little work, however, has been done on analyzing focusing characteristics of two-dimensional Subwavelength Planar Metallic Lenses (SPMLs) with the same structures as the SPDLs.

So, in this paper, we present the rigorous electromagnetic analysis and design of SPMLs based on concentric nano-rings with modulated width by using 3-D finitedifference time-domain method [16]. The focusing characteristics of planar lenses with nano-scale rings which have the same depth but tuning widths, for linear polarization illumination and for different material (metal sliver and dielectric silica) have been investigated and compared. The simulation results indicate that using the design method of SPDLs, SPMLs can indeed be employed in focusing light in the visible range. The comparative results have shown that when the thickness is less than $200 \mathrm{~nm}$, though SPMLs have opaque silver film and SPDLs are etched on the glass substrate without opaque parts, SPMLs will still support higher intensity focal spot with narrower Full-Width Half-Maximum (FWHM) beam width and longer depth of focus at the focal region since the propagation constants of SPPs and relative phases in metallic rings are strongly dependent on the rings' width and film's thickness. When the thickness is larger than $200 \mathrm{~nm}$, the loss of metal will increase, so SPDLs will have better focal performances than those of SPMLs. This ability to manipulate a beam of light in the visible range on the nano-scale metal rings can improve the quality of systems in applications such as photonic and plasmonic integrated devices, optical data storage, sensing and imaging, etc.

This paper is organized as follows: In Section 2 we discuss the basic formulas used in our study, and describe subwavelength structures of SPMLs. In Section 3 we provide and compare results of rigorous designs and analysis of SPMLs and SPDLs with different thicknesses in detail. Finally, a brief conclusion and our contribution are given in Section 4.

\section{Theoretical Background and Structure}

\subsection{Basic Formulas}

In our earlier work [15], to a conventional diffractive lens, we approximated its continuous profile to a piecewise-linear profile, and then encoded the individual linear segments as binary subwavelength structures, so a
Subwavelength Planar Dielectric Lens (SPDL) will be made. In a geometrical argument, the phase delay as a function of radius $r$ from the center of a lens can be obtained readily according to the equal optical length principle:

$$
\phi(r)=2 \pi n\left(f-\sqrt{f^{2}+r^{2}}\right) / \lambda_{0}
$$

where $\lambda_{0}$ is the wavelength of light in free space, $n$ the refractive index of air and $f$ is the focal length of a lens. According to this phase distribution, for the normal incidence, the lens will have a continuous surface-relief profile, and then this profile can be approximated by linearly increasing widths of a subwavelength planar feature. Although SPDLs using this technique can provide good focusing performances, they contain small subwavelength feature sizes and large aspect ratios (ratio of minimum feature height to width) that make them difficult to fabricate. So in this paper, we use this technique to make the profile (concentric rings with modulated width) of SPDLs and SPMLs, but the thickness of lens is less than a micrometer.

\subsection{Description of Subwavelength Structures}

A schematic of a subwavelength planar lens is shown in Figure 1, where $D$ is the diameter of the lens, and $t$ is the thickness of the planar lens. The material of lens can be silver film or silica dielectric film, and outside the lens is air. From the cross section profile along the $x-x$ plane (as shown in Figure 1(b)), we can see the lens consists of many Ag/Air/Ag waveguide (like a metal-insulator-metal (MIM) structure) array or Silica/Air/Silica waveguide

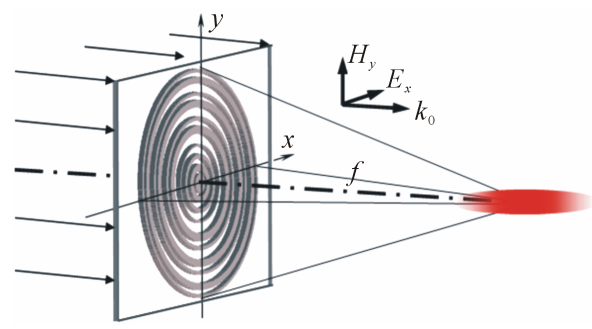

(a)

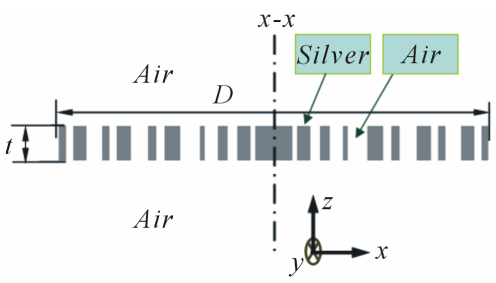

(b)

Figure 1. (a) A schematic of the investigated subwavlength planar metallic lens, and rings have different widths; (b) the cross section view along $x-x$ plane. 
array with fixed thickness and tuning width. Each individual silver ring's width is smaller than the incident wavelength; thus it can be treated as a plasmonic waveguide. As an incident electromagnetic wave meets the ring, it will be converted into SPPs mode, and transmitted along the waveguide, and then decoupled back to an electromagnetic mode with a phase delay upon exiting the ring (a lens's exiting plane). The complex propagation constant $\beta$, which is related to the phase change of the electromagnetic wave after it passes through the ring structure, can be expressed as $[17,18]$ :

$$
\begin{aligned}
& \tanh \left(\sqrt{\beta^{2}-\varepsilon_{d} k_{0}^{2}} \cdot w / 2\right) \\
& =-\left(\sqrt{\beta^{2}-\varepsilon_{m} k_{0}^{2}} / \sqrt{\beta^{2}-\varepsilon_{d} k_{0}^{2}}\right) \cdot\left(\varepsilon_{d} / \varepsilon_{m}\right)
\end{aligned}
$$

where $k_{0}=2 \pi / \lambda_{0}$ is wave vector of light in free space, and $\varepsilon_{m}$ and $\varepsilon_{d}=n_{d}^{2}$ are the relative dielectric constant of the metal medium and the dielectric inside the ring (in this study $\varepsilon_{d}=1$ for air), and $w$ is the ring width. The real and imaginary parts of the effective refractive index $n_{\text {eff }}$, defined to be $\beta / k_{0}$, determine the phase velocity and the propagation loss of the SPP modes, respectively. Besides, the previous researches have shown that the electric field relative phase at the center of the metal groove exit should be a function of groove depth [12]. So the dispersion relation between the effective refractive index, relative phase, and ring structures implies that the phase delay can be modulated by tuning both ring width and depth.

\section{Numerical Results and Discussion}

In this paper, the three-dimensional electromagnetic diffraction problem in a system of a planar lens is schematically shown in Figure 1. For SPMLs, we use silver, and for SPDLs, we use silica glass. The two sides of the lens is air. All of the simulation used the three-dimensional finite-difference time-domain method, and the boundary condition is set to the perfect matching layers. The wavelength of incident linear polarized light is 633 $\mathrm{nm}$, and in the visible light range, silver has a negative dielectric constant [19], and the glass has a positive dielectric constant. So the used metal is $\mathrm{Ag}$ with $\varepsilon_{m}=$ $-15.924+\mathrm{i} 1.076$, and the relative dielectric constant of the used dielectric $\mathrm{S}_{\mathrm{i}} \mathrm{O}_{2}$ is 2.126 at the wavelength of 633 $\mathrm{nm}$, respectively.

\subsection{Numerical Results}

The parameters of the lens are as follows: the diameter of the lens $\mathrm{D}=8 \mu \mathrm{m}$, the designed focal length $f=1.6 \mu \mathrm{m}$ and the thickness of rings $t=200 \mathrm{~nm}$. The maximum ring size and the minimum ring size are $600 \mathrm{~nm}$, and $50 \mathrm{~nm}$, respectively. Accordingly, the maximum air width and the minimum one are $300 \mathrm{~nm}$, and $60 \mathrm{~nm}$, respectively. In this study, we define the Depth of Focus (DOF) as a region over which the intensity is greater than $80 \%$ of the maximum intensity along the optical axis, and the focal shift $(\Delta f)$ can be defined as the value that denotes the difference between the position of the maximum irradiance position along the light axis and the geometric focal length $f(f=1.6 \mu \mathrm{m})$ defined as in Equation (1).

We demonstrate beam focusing by the SPML. The magnitude and profile of electric field intensity focused by the SPML is shown in Figure 2(a), which can give a global review of focusing characteristics for the SPML. A clear-cut focus appears about $1.88 \mu \mathrm{m}$ away from the exit surface, which is a little larger than the designed focal length $1.6 \mu \mathrm{m}$. This result clearly verifies the feasibility of the SPML. Furthermore, Figure 2(b) shows the profile of electric field intensity focused by the SPDL with the same structure sizes as the SPML, and the focal length is about $1.84 \mu \mathrm{m}$. Both the metallic lens and the dielectric lens can focus the light well. Figure 2(d) and (e) show the focal spot intensity along the real focal plane $(z=1.88 \mu \mathrm{m}$ for the SPML, and $z=1.84 \mu \mathrm{m}$ for the SPDL) of the metallic lens and the dielectric lens, respectively. In order to reveal the focusing performance of lenses more clearly, the electric field intensity along $\mathrm{z}$ $(x=y=0)$ direction (the optical axis), and along $x(y=0$, $\mathrm{z}$ is equal to the real focal position), for the SPDL and the SPML are shown in Figures 2(c) and (f), respectively. And DOF, the focal shift, FWHM and the Peak value of the SPDL are $0.47 \mu \mathrm{m}, 0.24 \mu \mathrm{m}, 0.42 \mu \mathrm{m}\left(0.66 \lambda_{0}\right)$, and 8.8 units, respectively. The same parameters for the SPML are $0.58 \mu \mathrm{m}, 0.28 \mu \mathrm{m}, 0.31 \mu \mathrm{m}\left(0.49 \lambda_{0}\right)$, and 18.8 units, respectively. It is worth noting that, although there are many opaque metal rings on the SPML, the peak intensity of the SPML at the focal plane is much higher than that of the SPDL (about 2.14 times), and also, the FWHM of the SPML is narrower than that of the SPDL $(0.31 \mu \mathrm{m}$ for the SPML, and $0.42 \mu \mathrm{m}$ for the SPDL, respectively). Both the metallic lens and the dielectric lens has the same profile structure and the same ring distribution which means two lenses have the same waveguide structures, but one is a MIM waveguide and the other is a dielectric waveguide. Two lenses are assumed to have $200 \mathrm{~nm}$ thickness, and the different thickness will produce different phase delay and the loss, so will have an important influence on the focal performances.

\subsection{Comparison of Results}

In order to make an insightful comparison between focusing behaviors of SPDLs and SPMLs with different coating thickness, the next simulations are carried out 

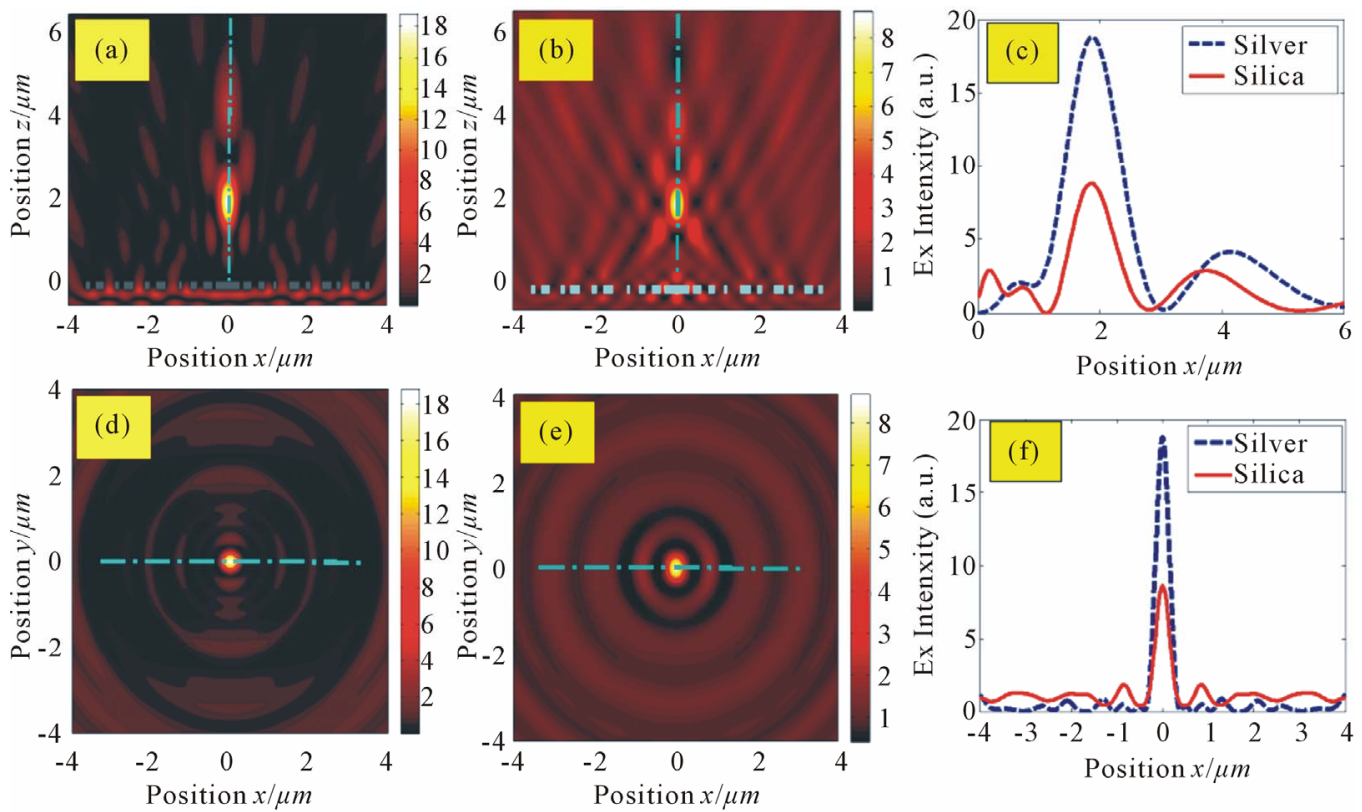

Figure 2. Electric field intensity distributions $|E x|^{2}$ for different subwavelength lenses: (a) For the SPML in $x z$ plane; (b) For the SPDL in $x z$ plane; (c) Axial $|E x|^{2}$ distributions along $z(x=y=0)$ direction; (d) For the SPML in $x y$ plane $(z=1.88 \mu \mathrm{m})$; (e) For the SPDL in $x y$ plane $(z=1.84 \mu \mathrm{m})$; and $(f)|E x|^{2}$ distributions along $x$ direction at the real focal plane for SPML and SPDL, respectively. The gray rectangular blocks drawn in (a) are the cross section of the SPML, and the cyan ones drawn in (b) are the cross section of the SPDL, respectively.

using $\mathrm{Ag}$ film and $\mathrm{SiO}_{2}$ film whose thicknesses change from 50 to $400 \mathrm{~nm}$, with $50 \mathrm{~nm}$ space. Figures 3(a) and (b) depict the axial $|\mathrm{Ex}|^{2}$ distributions along $z(x=y=0)$ direction, and transverse $|\mathrm{Ex}|^{2}$ distributions along $x$ direction at the real focal plane from SPMLs with various thickness of silver, respectively. And Figures 3(c) and (d) depict the axial $|\mathrm{Ex}|^{2}$ distributions and transverse $|\mathrm{Ex}|^{2}$ distributions along $x$ direction at the real focal plane from SPDLs with various thickness of silica, respectively. It is obviously seen that there are certain distinctions for different thickness and different material, respectively.

To denote these distinctions more clearly, relevant analysis parameters, such as DOF, the focal shift, FWHM, and the peak intensity have been plotted as a function of thickness, as shown in Figures 4(a)-(d). It is observed from Figure 4(a) that the deviation of DOF shows little librations with different film thickness, and DOF of silver lenses are larger than those of silica lenses, which means SPMLs are more suitable for high-precision optical alignment systems, etc. Figure 4(b) shows that when increasing film thickness, the focal shift will decrease and the real focal length will be close to the designed value. To these phenomena, we think, to both kinds of lenses, there are many subwavelength profiles on the silver film or the silica film, and they form many nano waveguide structures, where electromagnetic waves will produce a complex coupling effect among SPPs, diffracted evanescent waves and propagated electromag- netic waves. When we increase the thickness, the length of waveguide will also increase, so the focal shift will decrease. But for SPMLs, due to SPPs and loss of metal, when the thickness is larger than $250 \mathrm{~nm}$, the focal shift will increase a litter with increasing silver thickness. This can also explain the phenomenon in Figure 4(c), which shows that when increasing silica thickness, the peak intensity increases for SPDLs, but the peak intensity of SPMLs increases firstly, and when thickness is larger than $200 \mathrm{~nm}$, the peak intensity of SPDLs will decrease with increasing silver thickness. To SPMLs, when thickness is under a certain value, the loss of metal will be low, and the coupling effect among SPPs, evanescent waves and propagated waves will play a main role for lens performances, and get a better focusing behavior with higher peak value and a narrower FWHM (as shown in Figure 4(d)), but when the thickness is larger enough (for example larger than about $200 \mathrm{~nm}$ in this design case), the loss of metal will spoil performances of the lens, and the peak intensity will decrease. To SPDLs, the loss of silica in visible range can be neglected, and there are no SPPs also, so the coupling effect between evanescent waves and propagated waves will determine the final focusing performances of the lens [20]. When increasing silica thickness, the phase delay at the exit of the lens will be close to Equation (1), and the peak intensity will increase. But with a deeper waveguide, the aspect ratio will be larger, which is not easy to fabrication. 


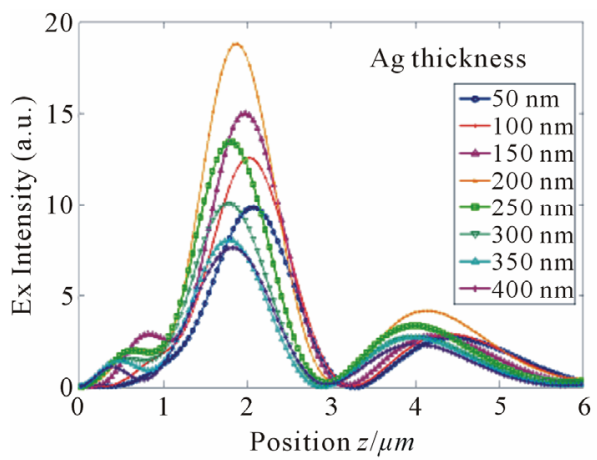

(a)

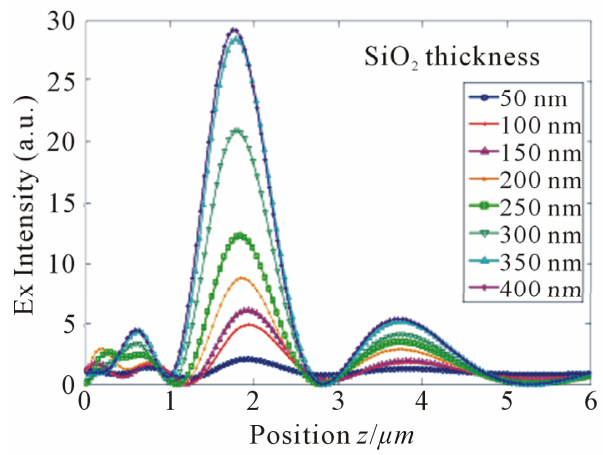

(c)

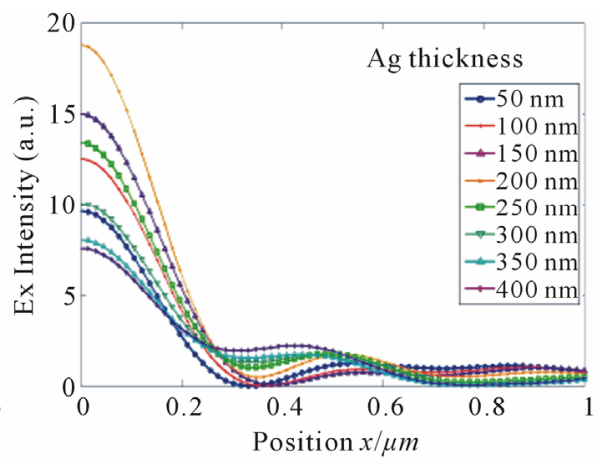

(b)

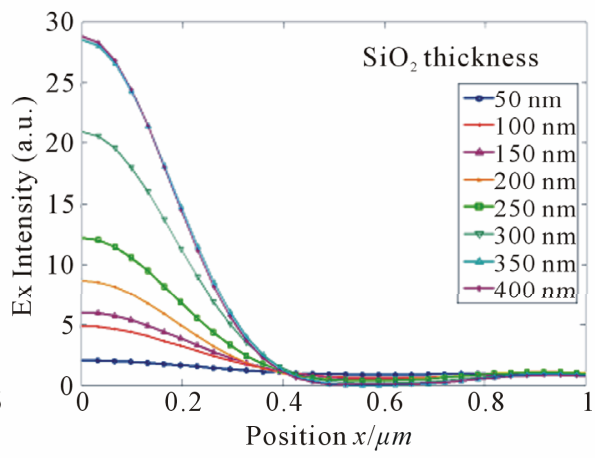

(d)

Figure 3. The electric field intensity distribution in cross sections of the focus alone the $z$ direction (a) and $x$ direction at the real focal plane (b), with different silver thickness for SPMLs, and the electric field intensity distribution in cross sections of the focus alone the $z$ direction (c) and $x$ direction at the real focal plane (d), with different silica thickness for SPDLs, under the illumination of $633 \mathrm{~nm}$ plane wave.
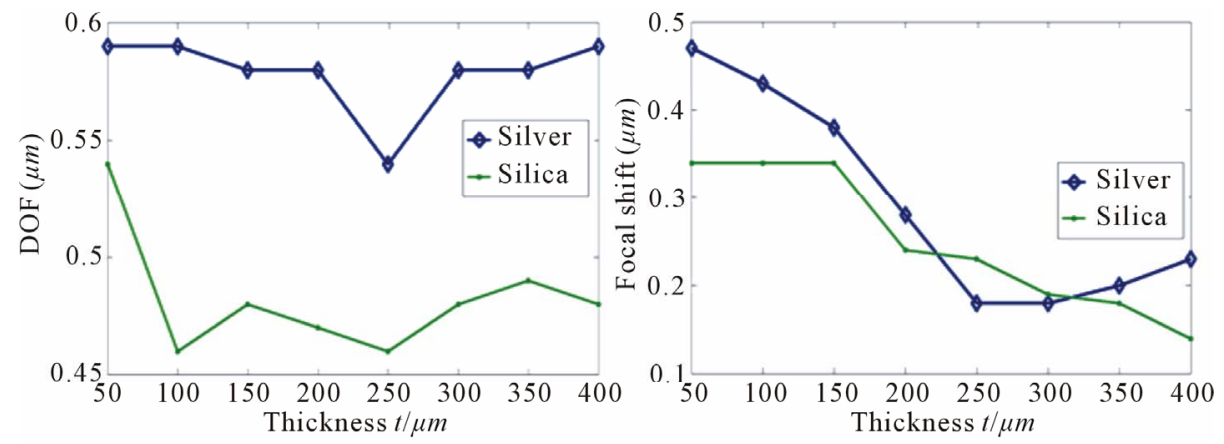

(a)

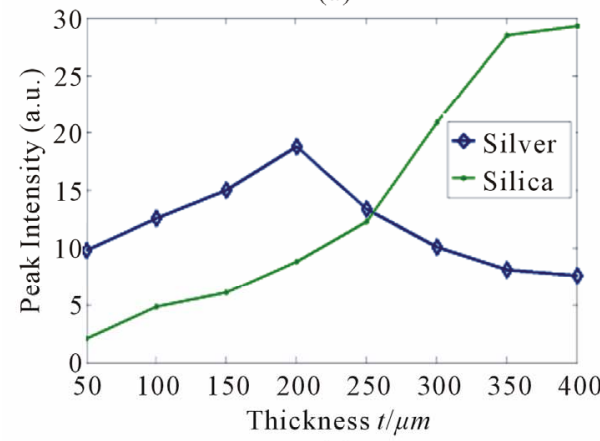

(b)

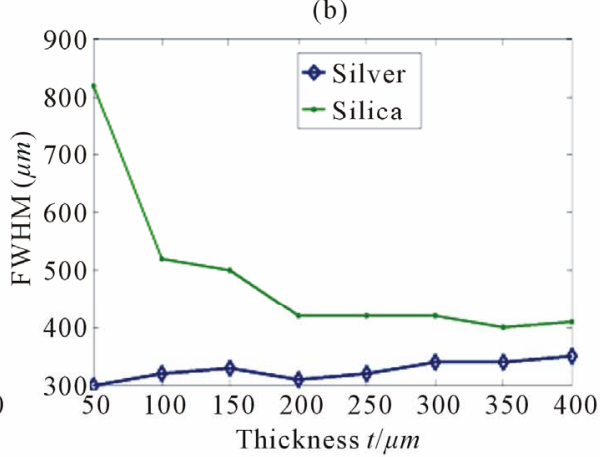

(d)

Figure 4. The focusing characteristics of SPDLs (Silica) and SPMLs (Silver) as a function of thickness: (a) DOF; (b) Focal shift; (c) Peak intensity; (d) FWHM. 
According to analysis results mentioned above, it is evident that SPMLs can indeed get better focusing performances and have the advantages of better spatial resolution (narrower FWHM), larger peak intensity, and longer DOF than SPDLs under certain lens thickness.

\section{Conclusion}

In conclusion, we have present a kind of Subwavelength Planar Metallic Lenses (SPMLs) to realize far-field optical focusing in the visible range. The SPML is designed based on the conventional Subwavelength Planar Dielectric Lens (SPDL), and has concentric rings with modulated width in the silver film. The focusing characteristics, such as DOF, the focal shift, FWHM, and the peak intensity, of both planar silver lenses and planar silica lenses, have been analyzed with the same rings structure and different film thickness. Compared with the SPDL that is no opaque coating, the SPML can support higher intensity focal spot, narrower full-width half-maximum beam width, and longer depth of focus at the focal region under certain lens thickness due to the coupling of SPPs, diffracted evanescent waves and propagated electromagnetic waves. It is clear that this kind of new SPMLs may have a good potential for applications in surface optical and optoelectronic integration systems, photonic and plasmonic integrated devices, etc.

\section{Acknowledgements}

This research is supported by Open Research Fund of State Key Laboratory of Millimeter Waves (K201217) and by Specialized Research Fund for the Doctoral Program of Higher Education (20091102120020).

\section{REFERENCES}

[1] T. W. Ebbesen, H. J. Lezec, H. F. Ghaemi, T. Thio and P. A. Wolff, "Extraordinary Optical Transmission through Subwavelength Hole Arrays," Nature, Vol. 391, 1998, pp. 667-669.

[2] L. Martin-Moreno, F. J. Garcia-Vidal, H. J. Lezec, K. M. Pellerin, T. Thio, J. B. Pendry and T. W. Ebbesen, "Theory of Extraordinary Optical Transmission through Subwavelength Hole Arrays," Physical Review Letters, Vol. 86, No. 6, 2001, pp. 1114-1117. doi:10.1103/PhysRevLett.86.1114

[3] L. Martin-Moreno, F. J. Garcia-Vidal, H. J. Lezec, A. Degiron and T. W. Ebbesen, "Theory of Highly Directional Emission from a Single Subwavelength Aperture Surrounded by Surface Corrugations," Physical Review Letters, Vol. 90, No. 16, 2003, pp. 167401-167403. doi:10.1103/PhysRevLett.90.167401

[4] W. Srituravanich, L. Pan, Y. Wang, C. Sun, D. B. Bogy and X. Zhang, "Flying Plasmonic Lens in the Near Field for High-Speed Nanolithography," Nature Nanotechno- logy, Vol. 3, 2008, pp. 733-737.

[5] H. J. Lezec, A. Degiron, E. Devaux, R. A. Linke, L. MartinMoreno, F. J. Garcia-Vidal and T. W. Ebbesen, "Beaming Light from a Subwavelength Aperture," Science, Vol. 297, No. 5582, 2002, pp. 820-822. doi:10.1126/science. 1071895

[6] J. H. Rice, "Beyond the Diffraction Limit: Far-Field Fluorescence Imaging with Ultrahigh Resolution," Molecular Biosystems, Vol. 3, No. 11, 2007, pp. 781-793. doi:10.1039/b705460b

[7] N. Fang, H. Lee, C. Sun and X. Zhang, "Sub-DiffractionLimited Optical Imaging with a Silver Superlens," Science, Vol. 308, No. 5721, 2005, pp. 534-537. doi:10.1126/science.1108759

[8] Y. Fu, W. Zhou, L. E. N. Lim, C. L. Du and X. G. Luo, "Plasmonic Microzone Plate: Superfocusing at Visible Regime," Applied Physics Letters, Vol. 91, 2007, pp. 61124-61126.

[9] R. G. Mote, S. F. Yu, B. K. Ng, W. Zhou and S. P. Lau, "Near-Field Focusing Properties of Zone Plates in Visible Regime-New Insights," Optics Express, Vol. 16, No. 13, 2008, pp. 9554-9564. doi:10.1364/OE.16.009554

[10] Y. Liu, Y. Q. Fu, X. L. Zhou, Z. W. Xu, F. Z. Fang and X. T. Hu, "Experimental Study of Indirect Phase TuningBased Plasmonic Structures for Finely Focusing," Plasmonics, Vol. 6, No. 2, 2011, pp. 227-233. doi:10.1007/s11468-010-9192-1

[11] H. F. Shi, C. T. Wang, C. L. Du, X. G. Luo, X. C. Dong and H. T. Gao, "Beam Manipulating by Metallic NanoSlits with Variant Widths," Optics Express, Vol. 13, No. 18, 2005, pp. 6815-6820. doi:10.1364/OPEX.13.006815

[12] H. F. Shi, C. L. Du and X. G. Luo, "Focal Length Modulation Based on a Metallic Slit Surrounded with Grooves in Curved Depths," Applied Physics Letters, Vol. 91, No. 9, 2007, pp. 9311-9313.

[13] L. Verslegers, P. B. Catrysse, Z. F. Yu, J. S. White, E. S. Barnard, M. L. Brongersma and S. H. Fan, "Planar Lenses Based on Nanoscale Slit Arrays in a Metallic Film," Nano Letters, Vol. 9, No. 1, 2009, pp. 235-238. doi: $10.1021 / \mathrm{nl} 802830 \mathrm{y}$

[14] Q. Chen, "A Novel Plasmonic Zone Plate Lens Based on Nano-Slits with Refractive Index Modulation," Plasmonics, Vol. 6, No. 2, 2011, pp. 381-385. doi:10.1007/s11468-011-9214-7

[15] D. Feng, Y. B. Yan, G. F. Jin and S. S. Fan, "Beam Focusing Characteristics of Diffractive Lenses with Binary Subwavelength Structures," Optics Communications, Vol. 239, No. 4-6, 2004, pp. 345-352. doi:10.1016/j.optcom.2004.05.050

[16] A. Taflove, "Computational Electrodynamics: The FiniteDifference Time-Domain Method," Artech House, Boston, 1995.

[17] Z. Sun and H. K. Kim, "Refractive Transmission of Light and Beam Shaping with Metallic Nano-Optic Lenses," Applied Physics Letters, Vol. 85, 2004, pp. 642- 644.

[18] R. Gordon and A. G. Brolo, "Increased Cut-Off Wavelength for a Subwavelength Hole in a Real Metal," Optics 
Express, Vol. 13, No. 6, 2005, pp. 1933-1938.

doi:10.1364/OPEX.13.001933

[19] E. D. Palik, "Handbook of Optical Constants of Solids II," Academic Press, Boston, 1991.

[20] D. Feng, N. F. Song, L. S. Feng, P. Ou and C. X. Zhang,
"Generation of an Extended Depth of Focus Using Diffractive Micro-Lenses with Binary Structures in the NonParaxial Domain," Journal of Optics A: Pure and Applied Optics, Vol. 11, No. 6, 2009, p. 65704.

doi:10.1088/1464-4258/11/6/065704 